http://www.esat.kuleuven.be/telemic/research.php

A new type of wearable antenna has been created as part of a project aiming at an integrated solution for monitoring, locating, alerting and communicating with senior citizens indoors

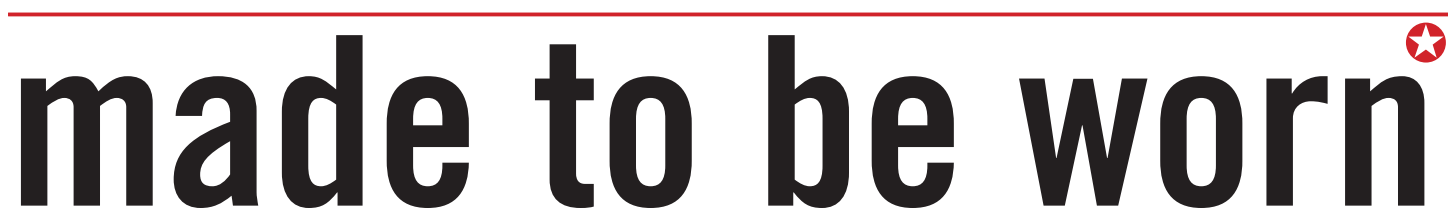

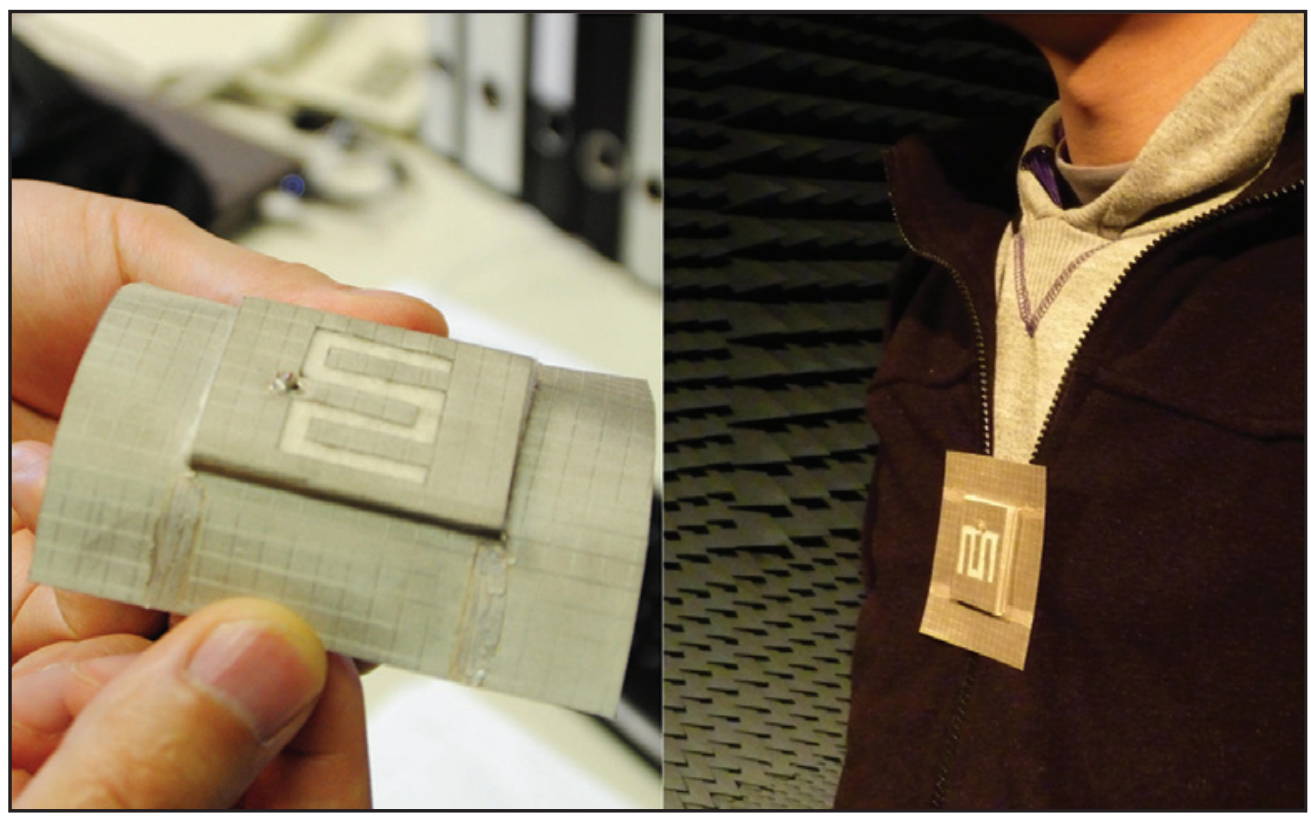

Researchers at Katholieke Universiteit Leuven and Universiti Malaysia Perlis are the first to have developed a fully textile waveguide antenna using a metamaterial inspired unit cell that is also used in composite right/left-handed transmission lines. The antenna is compact, robust and can be used for 2.45 and $5.4 \mathrm{GHz}$ dual-band WLAN applications.

\section{Different by design}

The integration of wireless sensing and communication into clothing is a very attractive solution in many sectors; for example, in the medical monitoring of hospitalised or homebound patients, or by emergency personnel in search and rescue missions, particularly in hazardous environments. Wearable, fabricbased antennas are light-weight, low-cost and unobtrusive compared to the usual rigid antenna structures. They have been in use for several years in the military sector but research is still ongoing worldwide in order to bring them into large scale consumer product use.

There are several challenges in the creation of body-worn antennas. The electromagnetic interaction between the human body and the antenna is a serious issue as: firstly, the irradiation of the human body over longer periods of time may present a health risk; and secondly, the body may strongly affect the performance of the antenna. The antenna's on-body behaviour,

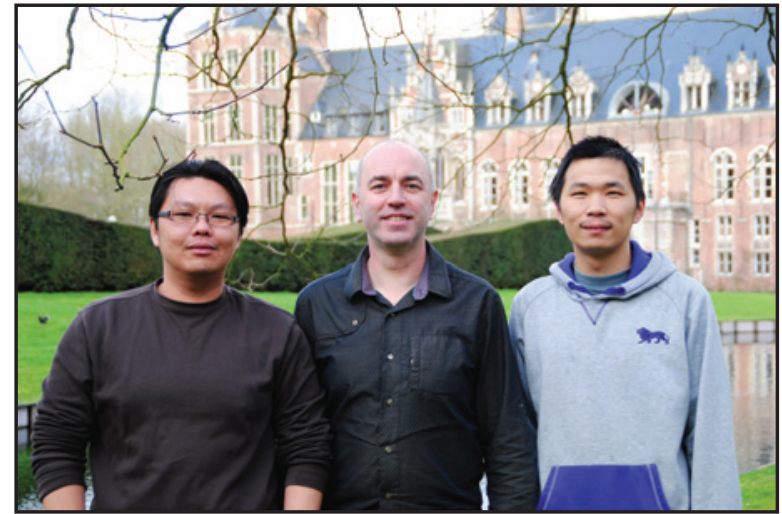

therefore, has to be properly understood. In addition, the properties of the textile materials used may not be readily available or may vary by manufacturer, and the antenna designs also need to take into account higher fabrication tolerances and show robustness against positional and directional changes when being worn.

\section{Cut from a different cloth}

The electrical size of an antenna depends on its physical dimensions and its operational frequency, and reducing the antenna size usually increases its operational frequency. If a metamaterial is used, its dispersion curve can be controlled by the design of the metamaterial itself, and this enables the creation of physically smaller radiators.

The essential feature of the antenna presented in this issue of Electronics Letters is the metamaterial inspired single cell embedded in the topology of the antenna, which allows the control of the field distribution within the antenna while keeping the overall design robust, simple and compact. The antenna, which is mounted on a felt substrate, radiates with similar nearfield distributions and radiation patterns through two different modes in two wireless frequency bands. Interference between the human body and the antenna is reduced by the use of a ground plane made from a commercially available shielding textile.

"This wearable textile antenna was developed within the framework of a project aiming at an integrated solution for monitoring, locating, alerting and communicating with senior citizens in an indoor environment," said Sen Yan, a researcher at Katholieke Universiteit Leuven and the first author of the Letter.

"The system is to be deployed in the form of a network of low-cost wireless sensors within a home. For real-time alerting in the event of emergencies it uses a stepped frequency continuous wave radar concept, combined with dedicated signal processing capabilities. The integration of an electronic module with the textile antenna, which contains several vitalsign sensors, a microcontroller and a wireless communication module, will complete the worn part of this system."

\section{Future systems}

Technical textiles are continuing to advance and this, combined with the rapid development of integrated circuit technology and the corresponding miniaturisation of electronics, according to Yan, makes it likely that wearable electronics and textile antennas will be combined within the next decade to form complete miniaturised wearable systems integrated into clothing.

"Sophisticated signal processing techniques, increased storage capabilities and high quality wireless connections will allow the design and manufacture of systems with unique high performance properties," he said. "Besides being able to increase productivity in specialised occupational segments, we would very much like to see this technology being widely applied in the healthcare sector, where it could result in an improved quality of life for many patients." 